\title{
Overexpressed EDIL3 predicts poor prognosis and promotes anchorage-independent tumor growth in human pancreatic
} cancer

\author{
Shu-Heng Jiang 1,2,*, Yang Wang ${ }^{1,2,4, *}$, Jian-Yu Yang ${ }^{3, *}$, Jun Li ${ }^{2, *}$, Ming-Xuan Feng ${ }^{5}$, \\ Ya-Hui Wang ${ }^{1,2}$, Xiao-Mei Yang ${ }^{2}$, Ping $\mathrm{He}^{2}$, Guang-Ang Tian², Xiao-Xin Zhang ${ }^{2}$, \\ Qing Li ${ }^{1,2}$, Xiao-Yan Cao ${ }^{2}$, Yan-Miao Huo ${ }^{3}$, Min-Wei Yang ${ }^{3}$, Xue-Liang $\mathrm{Fu}^{3}$, Jiao $\mathrm{Li}^{3}$, \\ De-Jun Liư ${ }^{3}$, Miao Dai ${ }^{7}$, Shan-Yun Wen', Jian-Ren Gu ${ }^{1,2}$, Jie Hong ${ }^{6}$, Rong Hua ${ }^{3}$, \\ Zhi-Gang Zhang', Yong-Wei Sun ${ }^{3}$ \\ ${ }^{1}$ Shanghai Medical College of Fudan University, Shanghai, P.R. China \\ ${ }^{2}$ State Key Laboratory of Oncogenes and Related Genes, Shanghai Cancer Institute, Ren Ji Hospital, School of Medicine, \\ Shanghai Jiao Tong University, Shanghai, P.R. China \\ ${ }^{3}$ Department of Biliary-Pancreatic Surgery, Ren Ji Hospital, School of Medicine, Shanghai Jiao Tong University, Shanghai, \\ P.R. China \\ ${ }^{4}$ Department of Urology, Shanghai No.5 People's Hospital, Fudan University, Shanghai, P.R. China \\ ${ }^{5}$ Department of Liver Surgery, Ren Ji Hospital, School of Medicine, Shanghai Jiao Tong University, Shanghai, P.R. China \\ ${ }^{6}$ Division of Gastroenterology and Hepatology, Ren Ji Hospital, Shanghai Institution of Digestive Disease, Key Laboratory \\ of Gastroenterology and Hepatology, Ministry of Health, State Key Laboratory of Oncogene and Related Genes, Shanghai \\ Jiao-Tong University School of Medicine, Shanghai, P.R. China \\ ${ }^{7}$ Department of Obstetrics and Gynecology, Shanghai Jiao Tong University Affiliated Sixth People's Hospital, Shanghai, P.R. \\ China \\ *These authors contributed equally to this work
}

Correspondence to: Yong-Wei Sun, e-mail: syw0616@126.com

Zhi-Gang Zhang, e-mail: zzhang@shsci.org

Rong Hua, e-mail: lordhuarong@sohu.com

Jie Hong, e-mail: jiehong97@gmail.com

Keywords: EDIL3, prognosis, tumor growth, anoikis, pancreatic cancer

Received: June 16, 2015

Accepted: November 28, 2015

Published: December 28, 2015

\section{ABSTRACT}

Epidermal Growth Factor-like repeats and Discoidin I-Like Domains 3 (EDIL3), an extracellular matrix (ECM) protein associated with vascular morphogenesis and remodeling, is commonly upregulated in multiple types of human cancers and correlates with tumor progression. However, its expression pattern and underlying cellular functions in pancreatic ductal adenocarcinoma (PDAC) remain largely unexplored. In current study, we observed that expression of EDIL3 was significantly up-regulated in PDAC compared with normal controls in both cell lines and clinical specimens. In addition, elevated EDIL3 expression was positively correlated with patients' TNM stage and T classification. Kaplan-Meier analysis indicated that high EDIL3 expression was significantly associated with shorter overall survival times in PDAC patients. Multivariate Cox regression analysis confirmed EDIL3 expression, age, lymph node metastasis and histological differentiation as independent prognostic factors in PDAC. Knockdown of EDIL3 showed no significant influence on cell viability, migration, invasion and starvation-induced apoptosis, but compromised anoikis resistance and anchorage independent tumor growth of PDAC cells. Meanwhile, treatment with recombinant EDIL3 protein markedly promoted anoikis resistance and anchorage independent tumor growth. Mechanistically, we demonstrated that altered protein expression of $\mathrm{Bcl}-2$ family might contribute to the oncogenic activities of EDIL3. In conclusion, this study provides evidences that EDIL3 is a potential predictor and plays an important role in anchorage independent tumor growth of PDAC and EDIL3-related pathways might represent a novel therapeutic strategy for treatment of pancreatic cancer. 


\section{INTRODUCTION}

Pancreatic ductal adenocarcinoma (PDAC) remains one of the most deadly malignancies with frequent metastasis and recurrence [1]. Most cases with pancreatic cancer are diagnosed in advanced stages and are ineligible for potentially curative resection, which lead to a poor prognosis with a 5 -year survival rate of $6 \%$ in all patients [2]. Although great endeavors have been made in early diagnosis, surgical resection and systemic chemotherapy for pancreatic cancer, the poor outcome of pancreatic cancer has remained unchanged since 40 years [3]. The disappointing survival rates for pancreatic cancer indicate the aggressive nature of this deadly disease. Therefore, to overcome this global challenging problem, a thorough understanding of the molecular mechanisms underlying the progression of pancreatic cancer is urgently needed $[4,5]$.

Extracellular matrix (ECM) proteins are made up of secreted molecules with implications in developmental patterning, stem cell niches and cancer [6]. Epidermal Growth Factor-like repeats and Discoidin I-Like Domains 3 (EDIL3), also known as developmentally regulated endothelial cell locus 1 (DEL-1), is a secreted (ECM) protein that composed of three epidermal growth factor (EGF) domains and two discoidin I-Like repeats [7]. EDIL3 is involved in the regulation of angiogenesis, cell attachment, and migration through an Arg-Gly-Asp (RGD) motif in its second EGF repeats by interacting with $\alpha_{v} \beta_{3}$ integrin [7-9]. EDIL3 is an embryonic endothelial cell protein and is not expressed after birth [7]. However, it has been demonstrated that EDIL3 was expressed in multiple types of cancer including breast cancer $[10,11]$, colon cancer [12], bladder cancer [13] and hepatocellular carcinoma [14-16]. Overexpressed EDIL3 can reduce tumor cell apoptosis and increase tumor vascularization, thus promoting tumor growth [10]. The capabilities of EDIL3 in tumor vascularization make it a candidate target for cancer anti-angiogenic therapy and also other diseases characterized by abnormal vascularization $[17,18]$. In colon cancer, down-regulation of EDIL3 inhibits tumor growth by antiangiogenesis and anti-proliferation [12]. In hepatocellular carcinoma, elevated autocrine EDIL3 protects cancer cells from anoikis through RGD-mediated integrin activation [15]. However, little is known about the expression pattern and cellular functions of EDIL3 in pancreatic cancer.

Anoikis is a specific form of apoptosis that occurs when cells detached from inappropriate ECM [19]. Acquisition of anoikis resistance is a critical process of metastasis by favoring cancer cells to survive during invasion and transference to the blood and lymph. Numerous potential regulators involved in anoikis of PDAC cells have been proposed, such as integrins and the anti-apoptotic $\mathrm{Bcl}-2$ family members [20]. EDIL3 is a well known ligand for $\alpha_{v} \beta_{3}$ integrin and activation of $\alpha_{v} \beta_{3}$ integrin can induce the expression of Bcl-2 in endothelial cell [21].

In the present study, we performed investigations to determine (a) the expression pattern and clinical significance of EDIL3 in pancreatic cancer, (b) the underlying roles of EDIL3 in pancreatic cancer cells, and (c) possible mechanism involved in EDIL3-mediated functions.

\section{RESULTS}

\section{EDIL3 mRNA and protein are significantly up-regulated in PDAC}

To illustrate the expression pattern of EDIL3 in PDAC, we firstly searched the mRNA expression level of EDIL3 in three GEO datasets. The results showed that EDIL3 expression was significantly up-regulated in PDAC tissues comparing with paired normal pancreatic tissues using GSE15471 (Figure 1A, $n=39, p=1.33 \mathrm{E}-11$ ) and GSE28735 (Figure 1B, $n=45, p=3.73 \mathrm{E}-8$ ). Expression of EDIL3 was also remarkably higher in the PDAC tissues than the normal pancreas as revealed by GSE16515 (Figure $1 \mathrm{C}, p=0.0008$ ). In present study, similar result was also observed in 32 paired PDAC and non-cancerous tissues as demonstrated by quantitative real-time PCR (Figure 1D, $n=32, p=0.0004$ ). To further address the protein change of EDIL 3 in PDAC tissues, Immunohistochemical analysis was performed in two independent PDAC tissue microarrays (TMA). In the commercial TMA (TMA1, OD-CT-DgPan01-006), we found that EDIL3 was significantly up-regulated in chronic pancreatitis (CHP) tissues and PDAC tissues compared with normal pancreas (NP) (Figure 1F). Importantly, EDIL3 immunoreactivity was specially distributed in PDAC cells except for islets. The representative staining of EDIL3 expression in NP, CHP as well as PDAC tissues were shown in Figure 1E and 1G. In TMA2, the expression of EDIL3 protein was also pronounced elevated in PDAC tissues and the pancreatic intraepithelial neoplasia-3 (PanIN3) compared with their normal counterparts (Figure 1H, $p=7.74 \mathrm{E}-69$ ).

\section{Relationship between EDIL3 expression and clinical parameters in patients with PDAC}

To determine the clinical significance of EDIL3 expression in PDAC, the Chi-square test was used to assess the relationships between EDIL3 protein expression and corresponding patients' clinicopathologic parameters including age, gender, tumor location, TNM stage, tumor size, T classification, lymph node metastasis, distant metastasis, vascular invasion and histological differentiation in TMA2. The results showed that EDIL3 expression in PDAC tissues was significantly correlated with TNM stage $(p=0.024)$ and T classification $(p=0.006)$, while no significant associations were observed between EDIL3 expression and age, gender, tumor location, tumor size lymph node metastasis, distant metastasis, vascular invasion and histological differentiation (Table 1). 
A

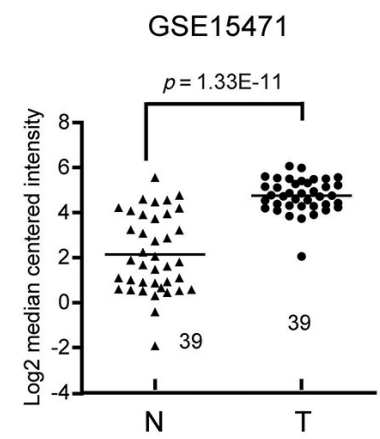

E

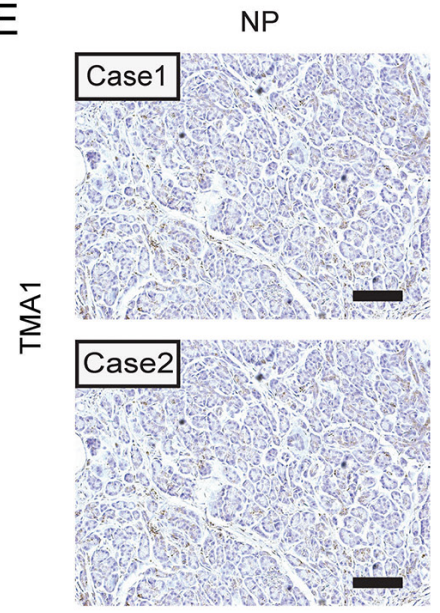

G

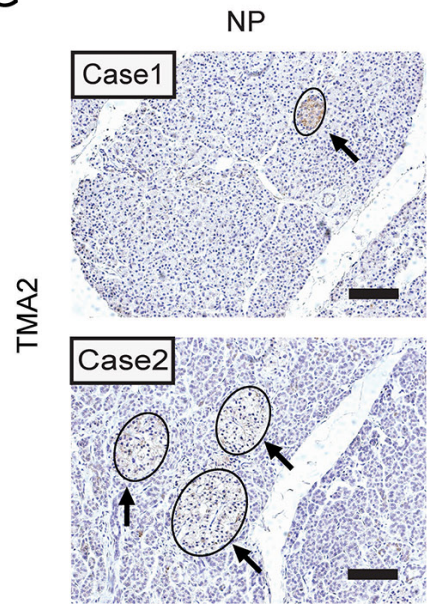

B

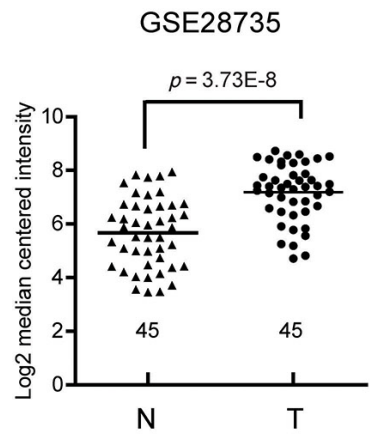

CP
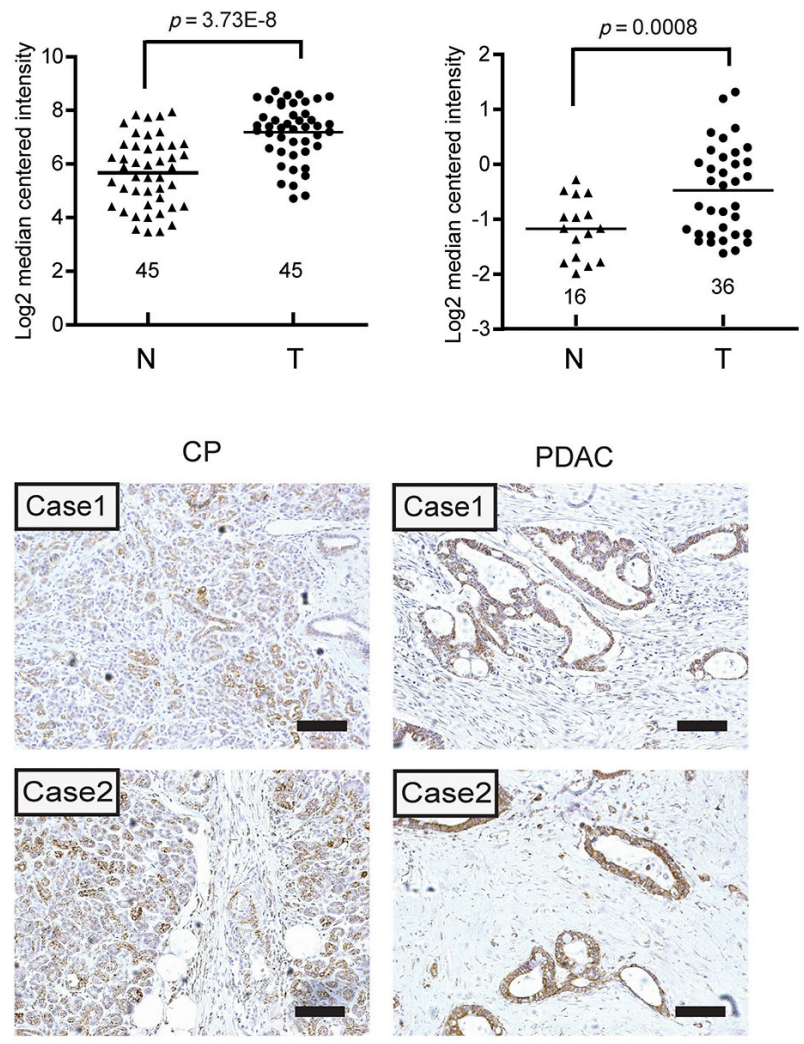

F

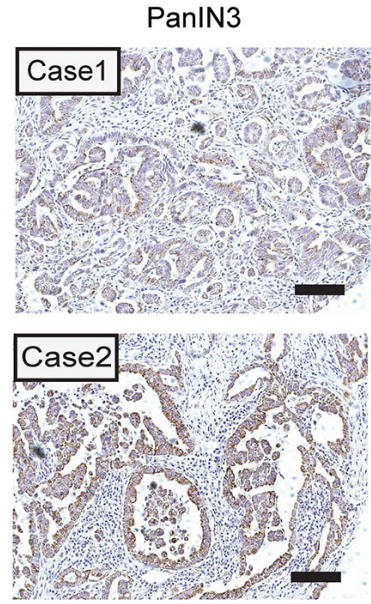

C

GSE16515

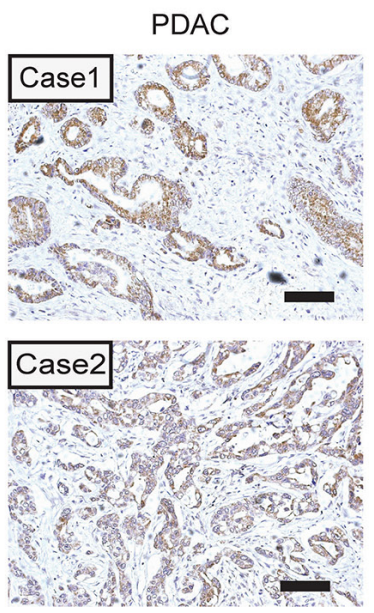

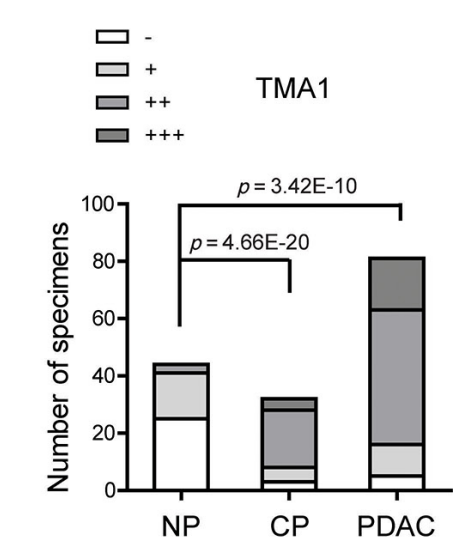

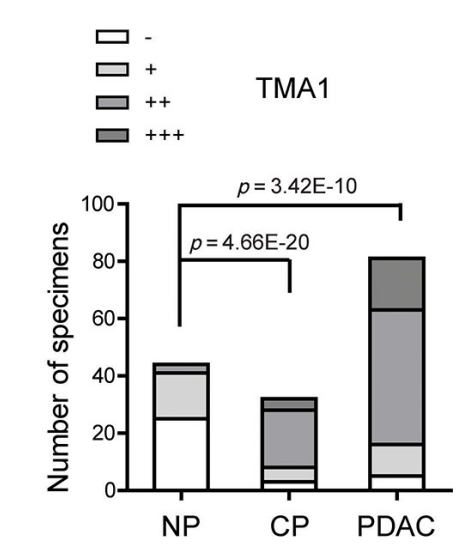

$\mathrm{H}$

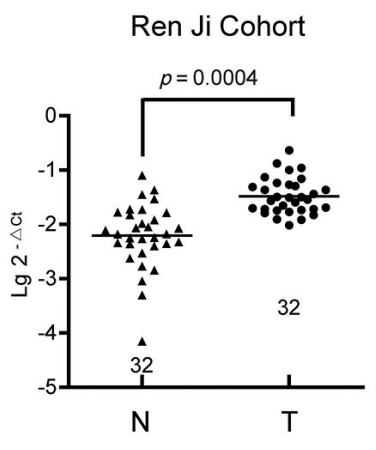

D

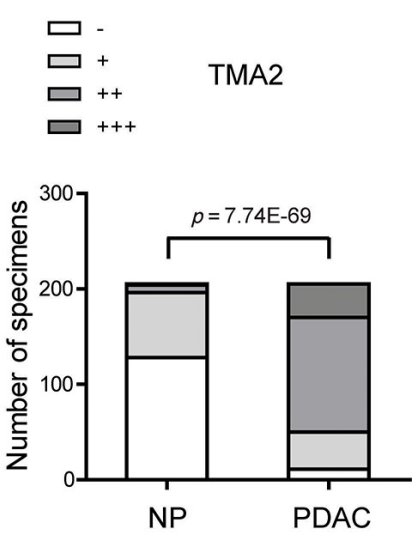

Figure 1: EDIL3 expression is increased in pancreatic cancer. (A) The mRNA expression of EDIL3 is upregulated in PDAC tissues $(\mathrm{T})$ compared with the normal pancreas tissues $(\mathrm{N})$ revealed using the GSE15471 dataset. (B) EDIL3 expression in the normal pancreas and PDAC tissues revealed by the GSE28735 dataset. (C) EDIL3 expression analysis in the normal pancreas and PDAC tissues in the GSE16515 dataset. (D) The mRNA expression level of EDIL3 in 32 matched tumor (T) and non-tumor tissue (N) derived from Ren Ji cohort was detected by Real-time quantitative PCR. (E) Representative photographs of the EDIL3 immunoreactivity in normal pancreas (NP), chronic pancreatitis (CP) and PDAC tissues in TMA1 (scale bar: $100 \mu \mathrm{m}$ ). (F) Comparisons of EDIL3 expression in TMA1 revealed by IHC analysis in NP, CP and PDAC tissues. (G) Representative photographs of the EDIL3 staining in NP, pancreatic intraepithelial neoplasia-3 (PanIN3) and PDAC tissues in TMA2. The arrows represent positive staining of EDIL3 in the islets (scale bar: $100 \mu \mathrm{m})$. (H) Comparisons of EDIL3 expression in TMA2 revealed by IHC analysis in NP, PanIN3 and PDAC tissues. 
Table 1: Correlations between EDIL3 expression and clinicopathologic parameters in patients with PDAC in TMA2

\begin{tabular}{|c|c|c|c|c|}
\hline \multirow[b]{2}{*}{$\begin{array}{c}\text { Clinicopathological } \\
\text { parameter }\end{array}$} & \multirow[b]{2}{*}{$\begin{array}{c}\text { Total } \\
205\end{array}$} & \multicolumn{2}{|c|}{ Expression of EDIL3 } & \multirow[b]{2}{*}{$p$ value } \\
\hline & & $\begin{array}{c}\text { Low } \\
(n=50, \%)\end{array}$ & $\begin{array}{c}\text { High } \\
(n=155, \%)\end{array}$ & \\
\hline \multicolumn{5}{|l|}{ Age (years) } \\
\hline$<65$ & 97 & $24(24.7)$ & $73(75.3)$ & 0.911 \\
\hline$\geq 65$ & 108 & $26(24.1)$ & $82(75.9)$ & \\
\hline \multicolumn{5}{|l|}{ Gender } \\
\hline Male & 117 & $29(24.8)$ & $88(75.2)$ & 0.879 \\
\hline Female & 88 & $21(23.9)$ & $67(76.1)$ & \\
\hline \multicolumn{5}{|l|}{ Tumor location } \\
\hline Head & 139 & $37(26.6)$ & $102(73.4)$ & 0.281 \\
\hline Body/tail & 66 & $13(19.7)$ & $53(80.3)$ & \\
\hline \multicolumn{5}{|l|}{ TNM (AJCC) } \\
\hline Stage I & 38 & $16(42.1)$ & $22(57.9)$ & 0.024 \\
\hline Stage II & 132 & $28(21.2)$ & $104(78.8)$ & \\
\hline Stage III & 21 & $5(23.8)$ & $16(76.2)$ & \\
\hline Stage IV & 14 & $1(7.1)$ & $13(92.9)$ & \\
\hline \multicolumn{5}{|l|}{ Tumor size } \\
\hline$\leq 2 \mathrm{~cm}$ & 27 & $9(33.3)$ & $18(66.7)$ & 0.246 \\
\hline$>2 \mathrm{~cm}$ & 178 & $41(23.0)$ & $137(77.0)$ & \\
\hline \multicolumn{5}{|l|}{ T classification } \\
\hline $\mathrm{T} 1,2$ & 42 & $17(40.5)$ & $25(59.5)$ & 0.006 \\
\hline $\mathrm{T} 3,4$ & 163 & $33(20.2)$ & $130(79.8)$ & \\
\hline \multicolumn{5}{|l|}{\begin{tabular}{|l|} 
Lymph node \\
metastasis \\
\end{tabular}} \\
\hline Absent & 136 & $38(26.6)$ & $98(73.4)$ & 0.096 \\
\hline Present & 69 & $12(19.4)$ & $57(80.6)$ & \\
\hline \multicolumn{5}{|l|}{ Distant metastasis } \\
\hline Absent & 191 & $49(25.7)$ & $142(74.3)$ & 0.120 \\
\hline Present & 14 & $1(7.1)$ & $13(92.9)$ & \\
\hline \multicolumn{5}{|l|}{ Vascular invasion } \\
\hline Absent & 178 & $45(25.3)$ & $133(74.7)$ & 0.446 \\
\hline Present & 27 & $5(18.5)$ & $22(81.5)$ & \\
\hline \multicolumn{5}{|l|}{\begin{tabular}{|l|} 
Histological \\
differentiation
\end{tabular}} \\
\hline Well & 11 & $4(36.4)$ & $7(63.6)$ & 0.468 \\
\hline Moderate/poor & 194 & $46(23.7)$ & $148(76.3)$ & \\
\hline
\end{tabular}

The bold number represents the $p$-values with significant differences. $P$ value was calculated by $\chi^{2}$ test or Fisher's exact test. 


\section{Up-regulated EDIL3 predicts poor prognosis of PDAC patients}

To evaluate the prognostic significance of EDIL3 in PDAC patients, the correlation between EDIL3 expression and corresponding clinical follow-up information were analyzed by Kaplan-Meier analysis and log-rank test. We first determined the prognostic value of EDIL3 at mRNA level using GSE28735. Three specimens without follow-up information were excluded from study. As shown in Figure 2A, patients with higher EDIL3 level (expression value $>8$ ) had significantly shorter survival time than those with a lower EDIL3 level (expression value $\leq 8$ ). At protein level, as demonstrated in TMA1, patients with higher EDIL3 expression had markedly decreased survival time than those with lower EDIL3 expression (Figure 2B, $p=0.0119$ ). To increase the statistic power of this result, we determined the prognostic value of EDIL3 in TMA2 with a total of 163 cases enrolled. And we found that high EDIL3 protein expression was remarkably associated with decreased overall survival (Figure 2C, $p=0.0036$ ). In addition, we determined the correlation between EDIL3 expression and overall survival in PDAC patients in early or advanced TNM stage and in the presence or absence of lymphatic metastasis. Kaplan-Meier analyses showed that overall survival was shorter in PDAC patients with higher EDIL3 expression regardless the state of TNM stage (Figure 2D) and lymphatic metastasis (Figure 2E). Furthermore, univariate and multivariate analyses were performed to identify the risk factor correlated with patients' prognosis in TMA2. Univariate Cox regression analyses showed that EDIL3 expression, age, TNM stage, tumor size, lymph node metastasis, distant metastasis and histological differentiation were significantly associated with overall survival (Table 2). Meanwhile, a multivariate Cox regression analysis identified that EDIL3 expression, age, lymph node metastasis and histological differentiation as
A

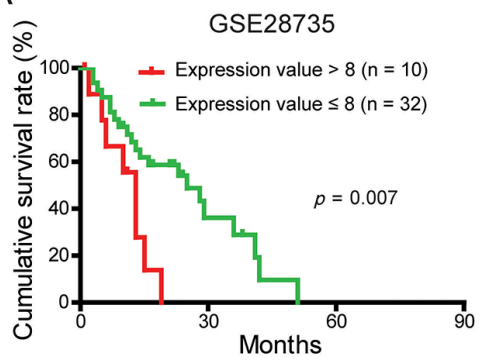

$\mathrm{B}$

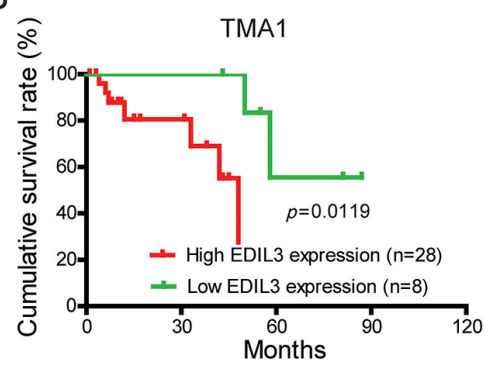

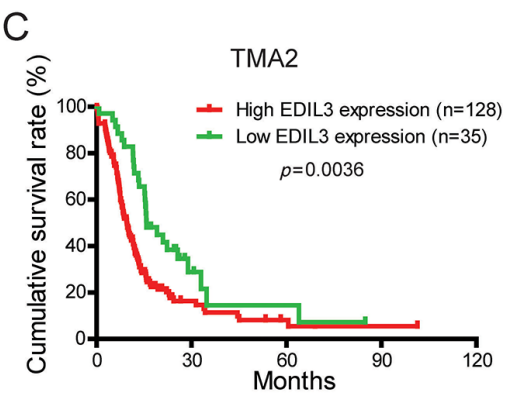
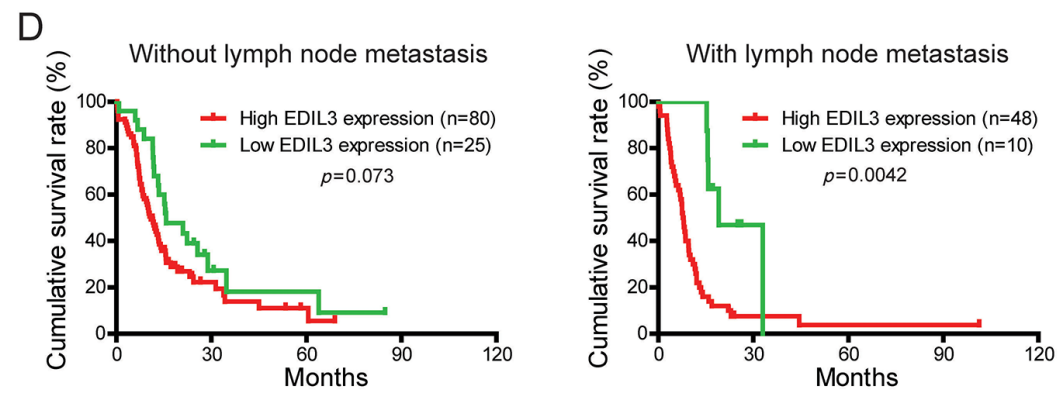

$E$
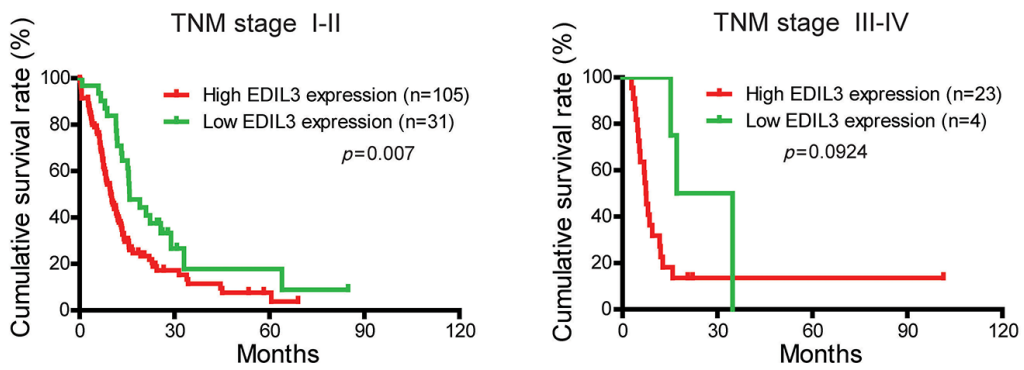

Figure 2: EDIL3 expression is correlated with overall survival rate independent of TNM stage and lymph node metastasis. (A) The correlation between EDIL3 expression and patient survival was conducted in GSE28735 dataset. (B) Overall survival analysis of PDAC patients with different EDIL3 protein expression in TMA1. (C) Overall survival analysis of PDAC patients with different EDIL3 protein expression in TMA2. (D) Comparison of overall survival in patients with or without lymph node metastasis was conducted based on EDIL3 expression. (E) Comparisons of overall survival between lower EDIL3 expression group and higher EDIL3 expression group in early TNM stage (I-II) cohort and in advanced TNM stage (III-IV) cohort. $P$ value was calculated by log-rank test. 
Table 2: Univariate and multivariate analysis of prognostic parameters for survival in patients with PDAC in TMA2

\begin{tabular}{|c|c|c|c|c|c|c|}
\hline \multirow{2}{*}{$\begin{array}{c}\text { Prognostic parameter } \\
\text { HR }\end{array}$} & \multicolumn{3}{|c|}{ Univariate analysis } & \multicolumn{3}{|c|}{ Multivariate analysis } \\
\hline & HR & $95 \% \mathrm{CI}$ & $p$ value & HR & $95 \% \mathrm{CI}$ & $p$ value \\
\hline Expression of EDIL3 (low vs. high) & 1.893 & $1.229-2.916$ & 0.004 & 1.717 & $1.106-2.663$ & 0.016 \\
\hline Age $(<65$ vs. $\geq 65)$ & 1.521 & $1.077-2.149$ & 0.017 & 1.836 & $1.270-2.654$ & 0.001 \\
\hline Gender (male vs. female) & 0.734 & $0.514-1.047$ & 0.088 & - & - & - \\
\hline Tumor location (head vs. body/tail) & 1.019 & $0.708-1.466$ & 0.920 & - & - & - \\
\hline TNM stage (I-II vs. III-IV) & 1.267 & $1.011-1.588$ & 0.040 & 0.930 & $0.648-1.334$ & 0.692 \\
\hline Tumor size $(\leq 2 \mathrm{~cm}$ vs. $>2 \mathrm{~cm})$ & 2.141 & $1.182-3.879$ & 0.012 & 1.773 & $0.971-3.238$ & 0.062 \\
\hline T classification $(\mathrm{T} 1,2$ vs. $\mathrm{T} 3,4)$ & 1.347 & $0.869-2.086$ & 0.182 & - & - & - \\
\hline $\begin{array}{l}\text { Lymph node metastasis (absent vs. } \\
\text { present) }\end{array}$ & 1.487 & $1.049-2.109$ & 0.026 & 1.688 & $1.133-2.515$ & 0.010 \\
\hline 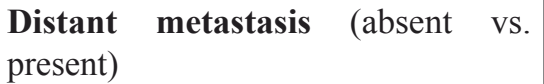 & 1.945 & $1.041-3.634$ & $\mathbf{0 . 0 3 7}$ & 2.195 & $0.844-5.705$ & 0.107 \\
\hline $\begin{array}{l}\text { Vascular invasion (absent vs. } \\
\text { present) }\end{array}$ & 1.579 & $0.969-2.572$ & 0.067 & - & - & - \\
\hline $\begin{array}{l}\text { Histological differentiation (well vs. } \\
\text { moderate/poor) }\end{array}$ & 2.475 & $1.011-6.058$ & 0.047 & 2.825 & 1.141 & 0.025 \\
\hline
\end{tabular}

HR: Hazard ratio; CI: Confidence interval. The bold number represents the $p$ value with significant differences.

independent predictors of the overall survival in patients with PDAC (Table 2). Taken together, these data above suggest that up-regulated EDIL3 predicts poor prognosis and might contribute to tumor progression in PDAC.

\section{EDIL3 inhibits anoikis and promotes anchorage- independent tumor growth in PDAC cells in vitro}

Given the close relationship between EDIL3 expression and poor clinical prognosis, we further determined the biological cellular functions of EDIL3 in PDAC cells. Consistent with the findings in PDAC tissues, EDIL3 expression was remarkably upregulated in all of six pancreatic cancer cell lines in relative to the nonmalignant hTERT-HPNE cells at both mRNA level (Figure 3A), protein level (Figure 3B) and secreted level (Figure 3C). Two PDAC cell lines with relatively higher EDIL3 expression, SW1990 and BxPC-3 cells, were selected for loss-of-function study. Stable expression of two short hairpin RNA (sh1, sh-2) targeting EDIL3 resulted in $>75 \%$ decrease in EDIL3 expression of SW1990 and BxPC-3 cells (Figure 3D). CCK-8 assay showed that there was no significant difference in cell viability between sh-Ctrl group and sh-EDIL3 group regardless of the presence (Supplementary Figure 1A) and absence (Supplementary Figure 1B) of 10\% fetal bovine serum. Transwell model was used to analyze the invasive potential. The result showed that knockdown of EDIL3 also failed to affect cell migration (Supplementary Figure 1C) or invasion (Supplementary Figure 1D) in SW1990 and BxPC-3 cells. Meanwhile, cell apoptosis assay and anoikis assay were performed by flow cytometric analysis. Indeed, cell apoptosis induced by serum deprivation for $48 \mathrm{~h}$ was not affected upon silencing of EDIL3 (Supplementary Figure 1E), which significantly inhibited anoikis resistance of SW1990 and BxPC-3 cells (Figure 3E). Consistent with this, we found caspase-3/7 activity in PDAC cells suspended in poly-Hema coated dishes for $48 \mathrm{~h}$ was markedly promoted by silencing of EDIL3 (Figure 3F). To further validate the role of EDIL3 in anoikis resistance, colony formation assay was performed. As shown in Figure $3 \mathrm{G}$, silencing of EDIL3 significantly reduced the anchorage-independent growth of SW1990 and BxPC-3 cells.

Next, a eukaryotic recombinant EDIL3 protein with high fidelity was generated as previously reported [15] (Figure 4A). As EDIL3 was critically involved in vascular morphogenesis and remodeling, we therefore studied the role of EDIL3 in PDAC angiogenesis. Cellconditioned medium (CM) of EDIL3-higher expression cells (SW1990 and BxPC-3 cells) significantly promoted the human umbilical vein endothelial cells (HUVECs) to develop more capillary-like structures compared with the CM of hTERT-HPNE cells (Figure 4B). Similarly, recombinant EDIL3 protein had no obvious implications 


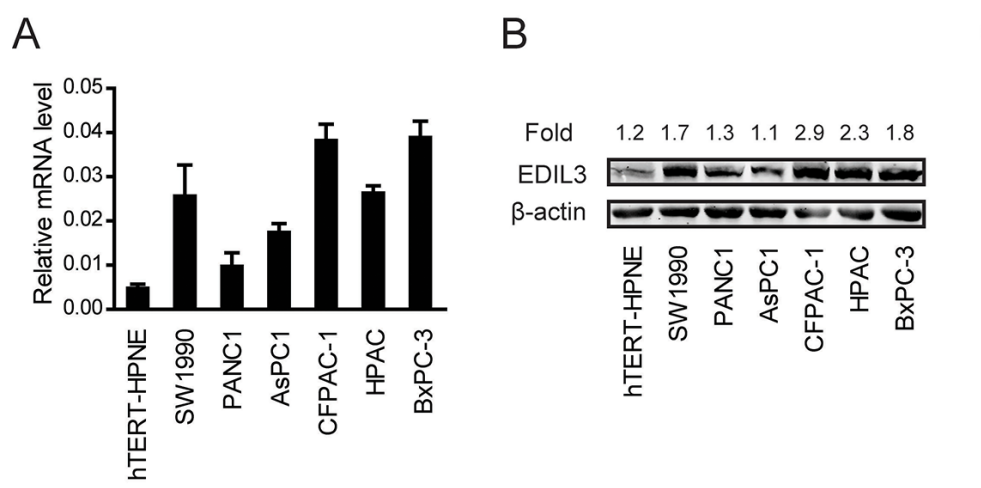

D

$E$
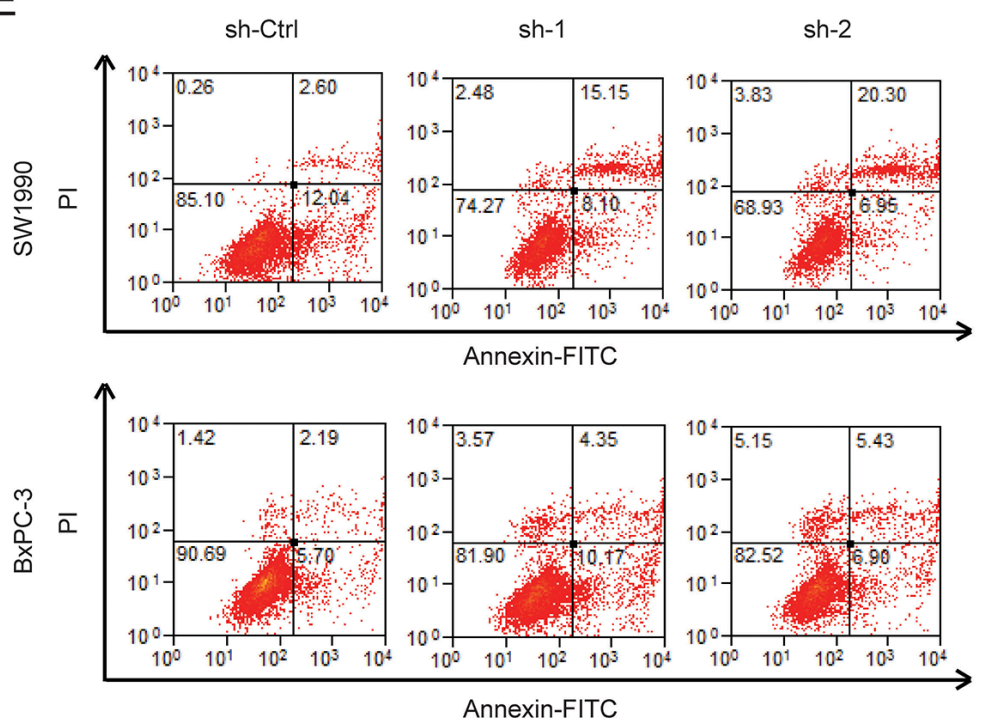
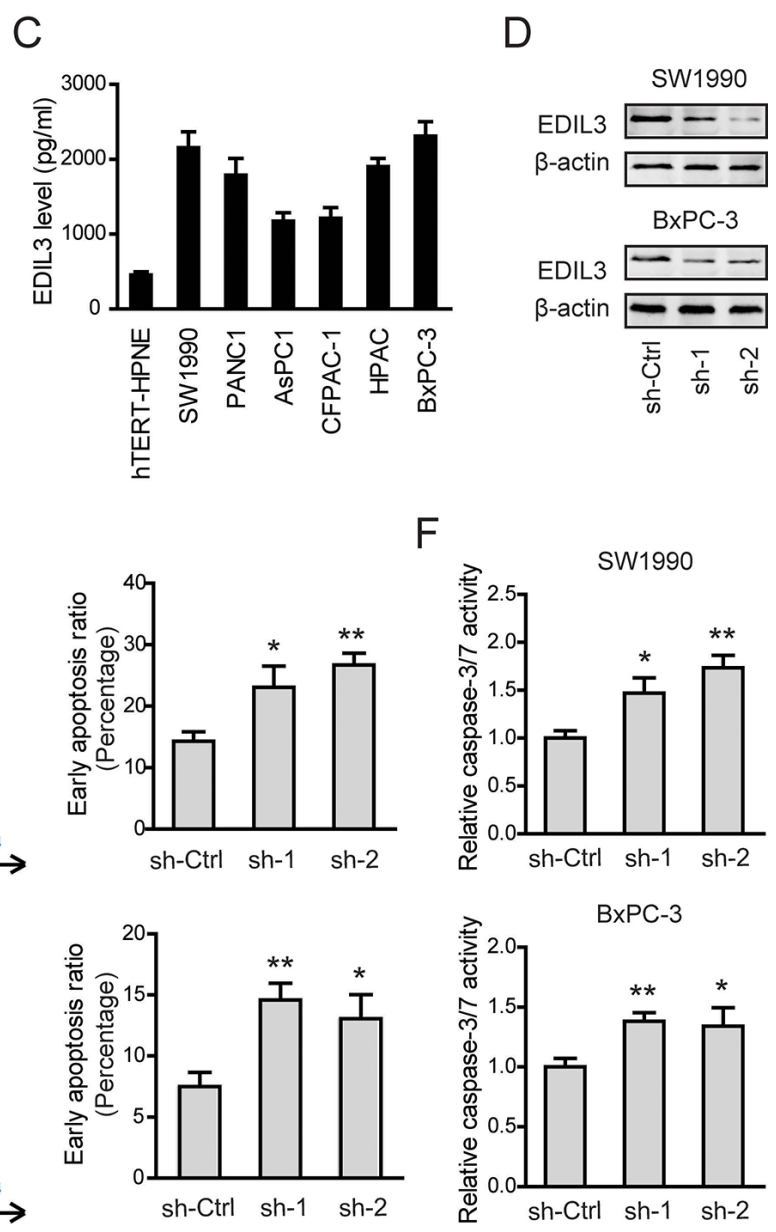

G
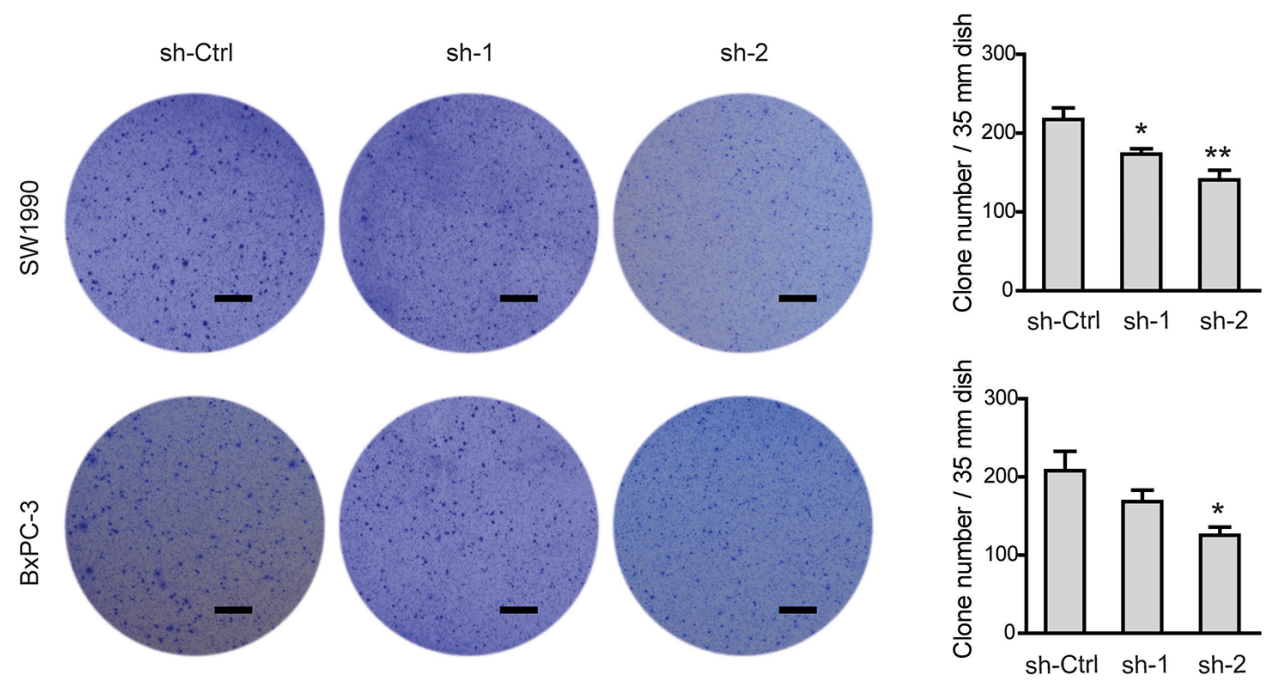

Figure 3: Knockdown of EDIL3 promotes anoikis and inhibits anchorage-independent tumor growth in PDAC cells. The mRNA (A), protein (B), secreted (C) levels of EDIL3 were assessed in six pancreatic cancer cell lines as well as a nonmalignant cell line hTERT-HPNE by quantitative real-time PCR, Western blotting and ELISA, respectively. (D) Interfere efficacy in SW1990 and BxPC-3 cell was detected by Western blotting. Knockdown of EDIL3 promoted anoikis as revealed by flow cytometry (E) and caspase-3/7 activity (F), and inhibited the colony formation ability (G) of SW1990 and BxPC-3 cells. Scale bar: $5 \mathrm{~mm}$. sh-Ctrl versus sh-1 or sh-2, ${ }^{*} P<0.05$, $* * P<0.01$. 


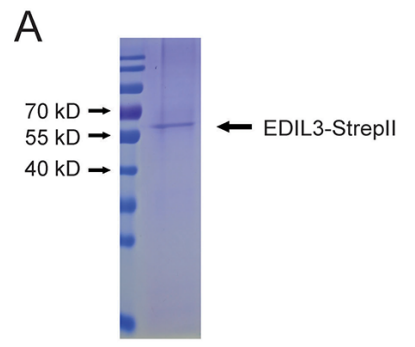

B
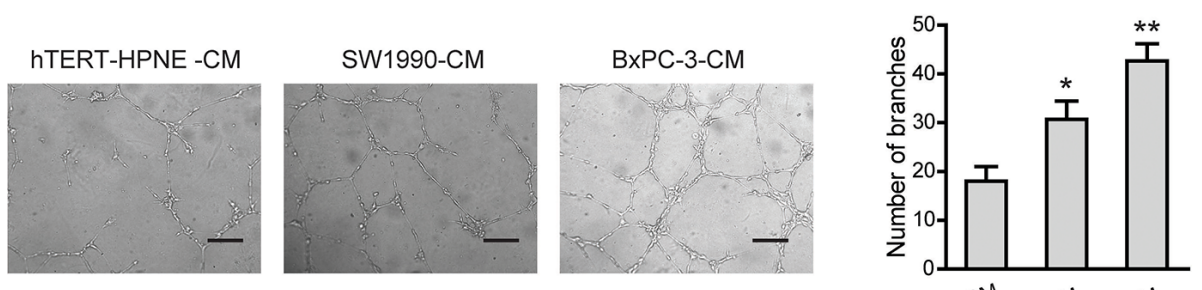

$$
\text { NTERT-HPNE-CM }{ }_{S W 1990}^{-C M}{ }_{B \times P C-3-C M}
$$

C
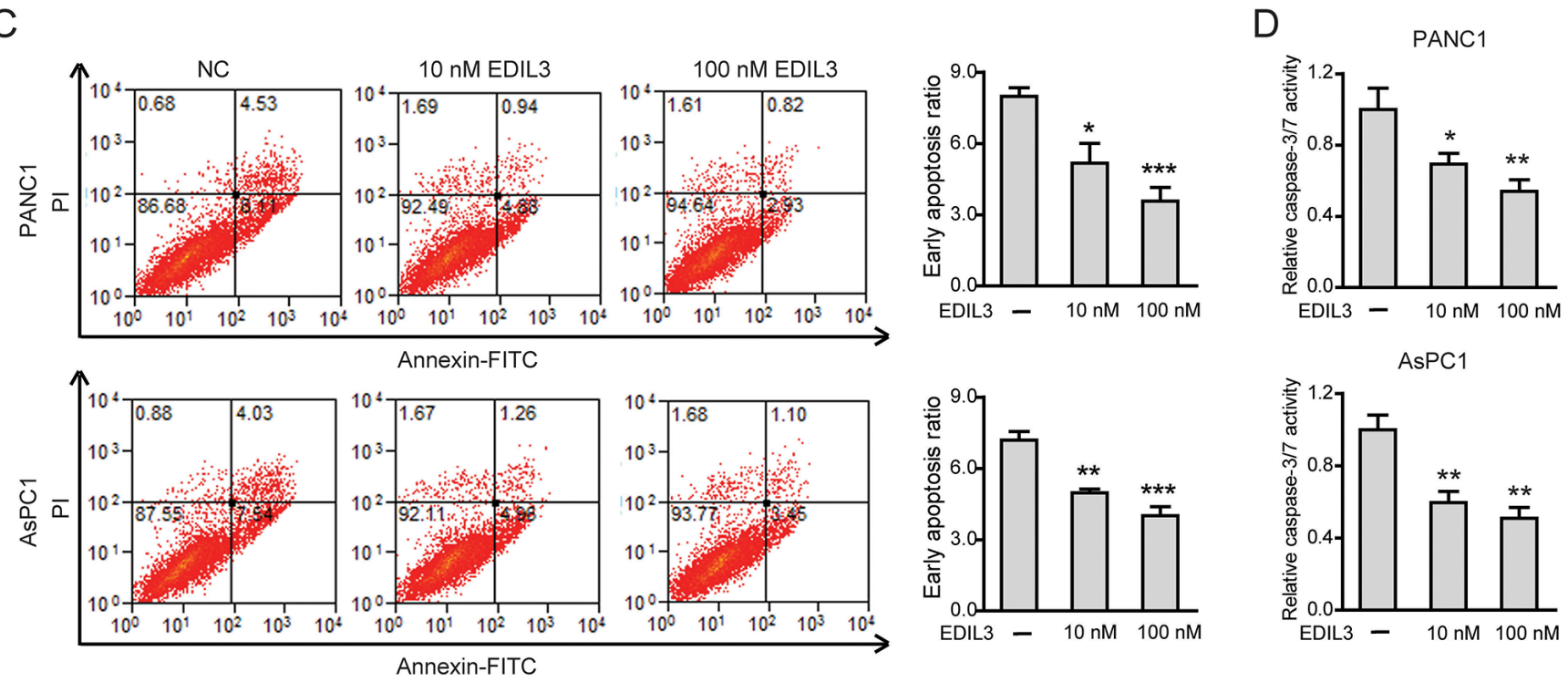

\section{$E$}
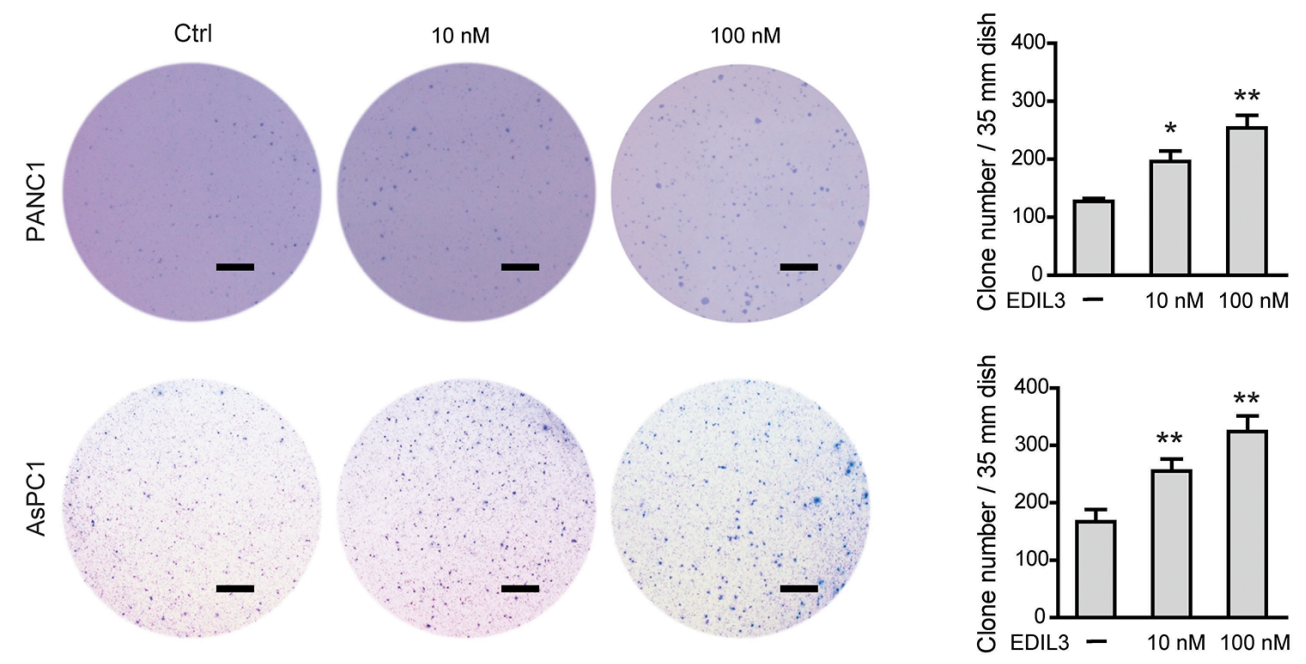

Figure 4: EDIL3 stimulation inhibits anoikis and promotes anchorage-independent tumor growth in PDAC cells. (A) Detection of purified recombinant human EDIL3 protein by coomassie blue staining. (B) PDAC cells derived EDIL3 promoted tumor angiogenesis in vitro. Scale bar: $200 \mu \mathrm{m}$. Treatment with recombinant EDIL3 protein inhibited anoikis as revealed by flow cytometry (C) and caspase-3/7 activity (D), and promoted the colony formation ability (E) of PANC1 and AsPC1 cells in a dose-dependent manner. Scale bar: $5 \mathrm{~mm}$. Ctrl versus $10 \mathrm{nM}$ EDIL3 or $100 \mathrm{nM}$ EDIL3, $* P<0.05,{ }^{*} P<0.01,{ }^{* * *} P<0.001$. 
on cell proliferation, migration, invasion and starvationinduced apoptosis (Supplementary Figure 2A-2E), whereas inhibited anoikis (Figure 4C, 4D) and promoted anchorage-independent tumor growth in a dose-dependent manner (Figure 4E). Collectively, these data indicate that EDIL3 promotes anoikis resistance and anchorageindependent growth and this prerequisite facilitates tumor progression.

\section{Knockdown of EDIL3 attenuates tumor growth in vivo}

To investigate EDIL3-mediated effects in vivo, a subcutaneous xenograft model was used (Figure 5A). Indeed, the tumor burden of PDAC in sh-EDIL3 group (sh-1 and sh-2) was significantly reduced compared with the sh-Ctrl group as revealed by tumor volume (Figure 5B) and tumor weight (Figure 5C). By immunohistochemical analysis, we found that EDIL3 expression in sh-EDIL3 group was markedly reduced compared with the shCtrl group (Figure 5D). Furthermore, the decreased expression of PCNA, a marker of cell proliferation, and increased cleaved caspase 3-positive cells were observed in sh-EDIL3 mice compared with those from sh-Ctrl mice (Figure 5E, 5F). Taken together, our data, as a proof of principle, demonstrate the promotive effect of EDIL3 on tumor growth in PDAC.

\section{EDIL3 correlates with altered expression of Bcl-2 family proteins}

To determine the molecular mechanism underlying EDIL3-mediated anchorage-independent growth of PDAC cells, we detected the effect of EDIL 3 on the expression
A

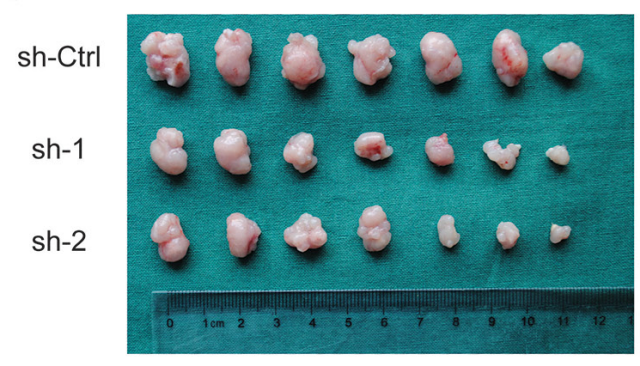

B

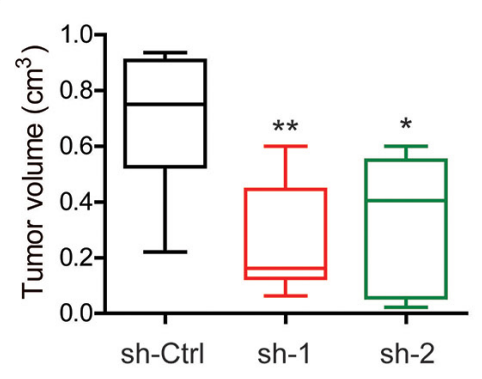

C

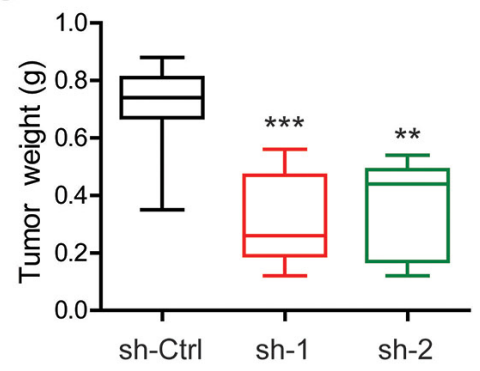

$\mathrm{E}$

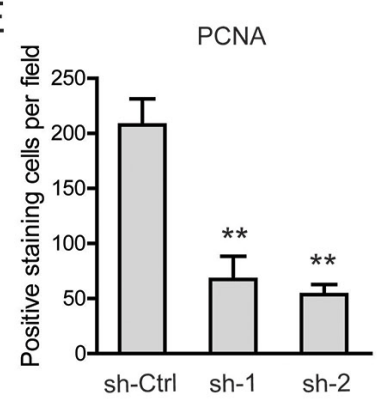

F

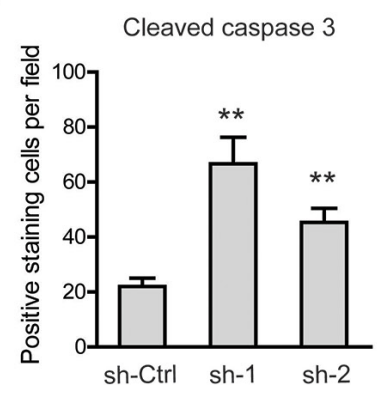

Figure 5: Knockdown of EDIL3 attenuates tumor growth in vivo. (A) Three weeks later, mice in sh-EDIL3 group showed relatively larger tumors compared with that in control group. (B) Tumor volume in sh-EDIL3 group was smaller than that control group $(n=7)$. (C) Tumor weight in sh-EDIL3 group was reduced compared with control group $(n=7)$. (D) Representative images of EDIL3, PCNA and cleaved caspase 3 in tissues from sh-EDIL3 and sh-Ctrl mice. Compared with sh-Ctrl mice, decreased expression of PCNA (E) and increased expression of cleaved caspase 3 (F) was observed in the tissue samples of sh-EDIL3 mice. Scale bar: $100 \mu \mathrm{m}$. sh-Ctrl versus sh-1 or sh- $2, * P<0.05, * * P<0.01, * * * P<0.001$. 
of Bcl-2 family proteins, including Bcl-2, Bcl-xL and Bax, which have been demonstrated to be important for tumor growth and anoikis resistance [22, 23]. As shown in Figure 6A, knockdown of EDIL3 reduced the protein level of Bcl-2 and Bcl-xL. Upon treatment with recombinant EDIL3 protein, $\mathrm{Bcl}-2$ and $\mathrm{Bcl}-\mathrm{xL}$ were significantly increased (Figure $6 \mathrm{~B}$ ). The pro-apoptotic protein, Bax, was faintly influenced by knockdown or introduction of EDIL3. To further confirm this result, we determined the correlation between EDIL3 and Bcl-2 expression by immunohistochemical analysis. As shown in Figure 6C-6D, EDIL3 protein levels in PDAC tissues were positively correlated with the expression levels of Bcl-2 $(r=0.399, p<0.001)$. Treatment with a specific inhibitor of Bcl-2, ABT-199 (also known as Venetoclax) at $1 \mu \mathrm{M}$, EDIL3-mediated anoikis resistance (Figure 6E) and anchorage-independent tumor growth (Figure 6F) were completely blocked. Taken together, this result indicates that altered expression of Bcl-2 proteins might involve in the oncogenic activities of EDIL3 in PDAC cells.

\section{DISCUSSION}

The current study focused on EDIL3, a well studied cellular protein whose clinical significance and underlying biological functions in PDAC remains unknown. We observed that EDIL3 was commonly up-regulated in both PDAC cell lines and clinical specimens compared with the normal controls. By silencing of EDIL3 expression and treatment with recombinant EDIL3 protein, we demonstrated that EDIL3 conferred PDAC cells with advantages of anoikis resistance and anchorage-independent growth through altering expression of Bcl-2 proteins. Our
A

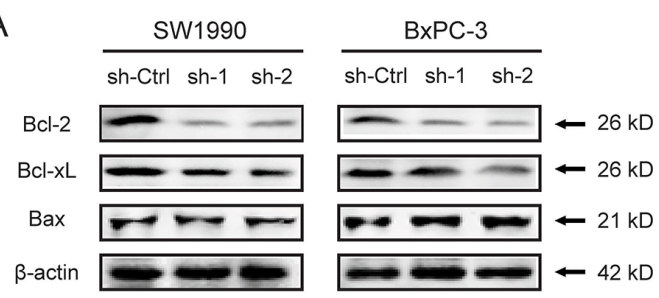

C
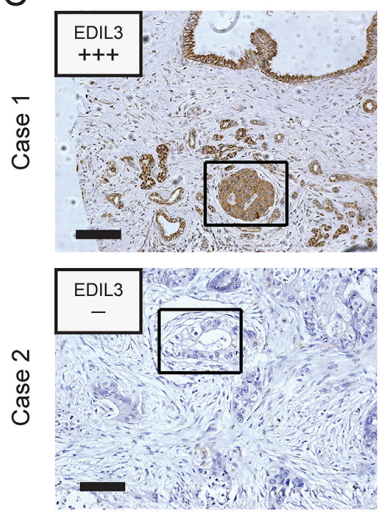

$\mathrm{E}$

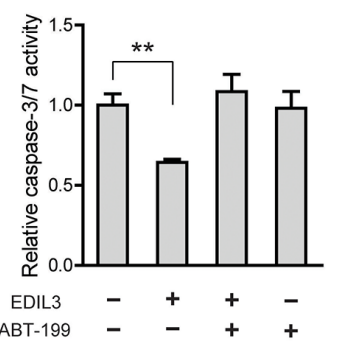

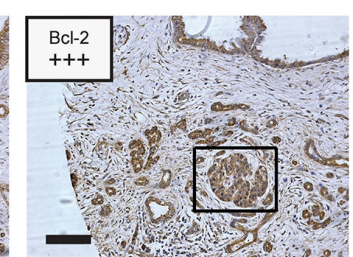

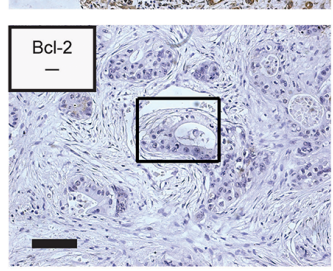

$\mathrm{F}$

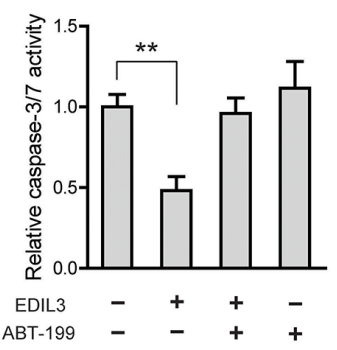

B

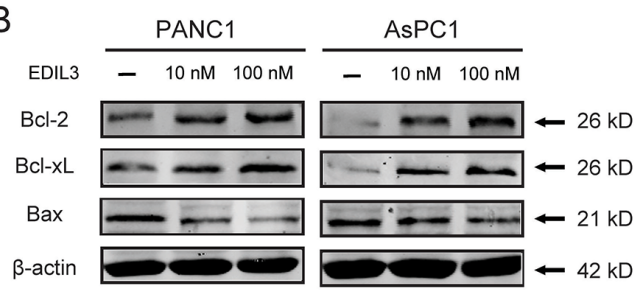

D

Correlation between EDIL3 and Bcl-2

\begin{tabular}{ccccc}
\hline \multirow{2}{*}{ IHC grade } & \multicolumn{4}{c}{ EDIL3 } \\
\cline { 2 - 5 } & $\begin{array}{c}- \\
(n=5, \%)\end{array}$ & $\begin{array}{c}+ \\
(n=11, \%)\end{array}$ & $\begin{array}{c}++ \\
(n=47, \%)\end{array}$ & $\begin{array}{c}+++ \\
(n=18, \%)\end{array}$ \\
\hline$-(n=12)$ & $3(60.0)$ & $2(18.2)$ & $6(12.8)$ & $1(5.6)$ \\
$\frac{1}{0}+(n=21)$ & $1(20.0)$ & $5(45.5)$ & $13(27.7)$ & $2(11.1)$ \\
$\frac{1}{\infty}++(n=31)$ & $1(20.0)$ & $3(27.3)$ & $20(42.6)$ & $7(38.9)$ \\
$+++(n=17)$ & $0(0.0)$ & $1(9.1)$ & $8(17.0)$ & $8(44.4)$ \\
\hline
\end{tabular}

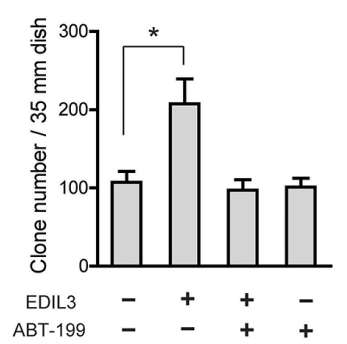

Figure 6: EDIL3 correlates with altered expression of Bcl-2 family proteins. Altered protein expression level of Bcl-2, Bcl-xL and Bax was detected upon knockdown of EDIL3 (A) or treatment with recombinant EDIL3 protein (B) (C) IHC analysis showed representative positive (up) and negative (down) staining of EDIL3 and Bcl-2 in consecutive sections. Indicated areas were marked by a square. Scale bar: $100 \mu \mathrm{m}$. (D) Statistical analysis of the correlation between EDIL3 and Bcl-2 expression in TMA1. $P$ values were calculated by the Spearman rank correlation test. In the presence of $1 \mu$ M ABT-199, the effects of recombinant EDIL3 protein (100 nM) on caspase-3/7 activity (E) and colony formation ability (F) was measured. 
present findings indicate that EDIL3-related pathway plays an important role in progression of human pancreatic cancer.

In human tissues, EDIL3 expression was identified restricted to endothelial cell in embryo but not adult [7]. Through binding to $\alpha_{v} \beta_{3}$ integrin, EDIL3 regulates endothelial cell attachment and migration as well as inflammatory cell recruitment and engulfment of apoptotic cell [24-26]. Consistent with the findings in hepatocellular carcinoma, EDIL3 expression was up-regulated during malignant transformation in PDAC $[14,15]$. In current study, however, EDIL3 expression was also observed in normal pancreas. This may explained by that these normal pancreas tissues were mainly non-tumor tissues derived from patients with PDAC and the tumor microenvironment of PDAC was likely to turn on the expression of EDIL3 in adjacent normal tissues. Previous reports have demonstrated that EDIL3 expression was induced by tumor-derived factors VEGF and p53 could regulate its transcription in primary endothelial cells $[27,28]$. And recently it has also been reported that EDIL3 was regulated by miR-137 in hepatocellular carcinoma [16]. Despite the critical roles of VEGF and the frequently mutation of p53 in pancreatic cancer, whether this type of modulation contributes to elevated EDIL3 expression remains further investigation [29-33]. Meanwhile, EDIL3 immunoreactivity was exclusively distributed in tumor cells, but not any positive staining in other cells except islet cells, such as immune cell and stromal cell, suggesting that EDIL3 might constitute an autocrine network in regulating tumor progression in PDAC and indicating that elevated EDIL3 expression might associate with prognosis of PDAC patients. Inconsistent with our findings, Bijlsma et al. have reported the immunoreactivity of EDIL3 in the stroma of pancreatic cancer [34]. However, the representative figures of EDIL3 staining in their paper also showed intense immunoreactivity of EDIL3 in the PDAC cells. This discrepancy may be due to the different clinical specimens analyzed or the different EDIL3 antibodies used.

To address the prognostic value of EDIL3 in PDAC, we performed Kaplan-Meier survival analyses and found that elevated expression of EDIL3 protein was inversely associated with clinical outcomes of PDAC patients. Meanwhile, the univariate and multivariate Cox regression analyses indicated that the up-regulated EDIL3 expression might be a risk factor for the overall survival of PDAC patients. This observation was consistent with the previous finding in hepatocellular carcinoma that elevated EDIL3 was an indicator for the poor prognosis [14, 15].

It has been demonstrated that EDIL3 can accelerate tumor growth by enhancing vascular formation and inhibiting tumor cell apoptosis in osteosarcoma and Lewis lung carcinoma [10]. Inconsistent with this, our data revealed that EDIL3 did not show significant implications on anchorage-dependent growth of pancreatic cancer cells. And in line with our previous observations in hepatocellular carcinoma [15], EDIL3 promoted anoikis resistance and anchorage-independent growth of pancreatic cancer cells. This type of difference may be induced by different mechanisms involved in EDIL3-mediated cellular functions in specific tumors. In hepatocellular carcinoma, EDIL3 exhibits its roles through activation of FAK-Src-Akt signaling by interacting with $\alpha_{v} \beta_{3}$ integrin. Apart from triggering an integrin-mediated phosphorylation of FAK-Src, Shc and MAPK, EDIL3 also acts as a survival factor via $\alpha_{v} \beta_{3}$ integrin in endothelial cell [35]. Through inducing expression of the anti-apoptotic protein, Bcl-2, activation of $\alpha_{\mathrm{v}} \beta_{3}$ opposes apoptosis of endothelial cell [21]. Consistent with this, treatment with recombinant EDIL3 protein markedly promoted the level of anti-apoptotic protein Bcl-2 and Bcl-xL, and inhibitor of Bcl-2 can completely abolish EDIL3-mediated oncogenic functions. Therefore, the mechanism by which EDIL3 promotes anoikis resistance and anchorage-independent growth may be due, in part, to the alternation of Bcl-2 family protein, which would protect the detached PDAC cells from anoikis.

In conclusion, we describe EDIL3 as a crucial factor in the control of anoikis resistance, anchorage-independent growth and clinical outcomes during human PDAC. Furthermore, we find that EDIL3 exerts its anti-apoptotic function by altering the protein expression of Bcl-2 family. This newly identified EDIL3/Bcl-2 axis might provide a further insight into the pathogenesis of pancreatic cancer and indicate a novel approaches that can be used for the treatment of pancreatic cancer.

\section{MATERIALS AND METHODS}

\section{Clinical samples}

Human pancreatic cancer tissue microarrays (TMA1, OD-CT-DgPan01-006) containing 81 cases of pancreatic ductal adenocarcinoma (PDAC), 44 cases of normal pancreas tissues and 32 cases of chronic pancreatitis tissues were purchased from Shanghai Outdo Biotech Inc. A total of 32 freshly frozen PDAC tissues and corresponding non-cancerous tissues were obtained from Ren Ji Hospital, School of Medicine, Shanghai Jiao Tong University between January 2012 and December 2013. TMA2 containing 205 pancreatic cancer specimens and corresponding non-cancerous tissues were also obtained from Ren Ji Hospital from January 2002 to June 2013. The histology and clinical stages were classified according to the seventh edition of the American Joint Committee on Cancer (AJCC) staging system. None of the patients had received radiotherapy, chemotherapy, hormone therapy or other related anti-tumor therapies before surgery. All the patients were provided with written informed consent 
before enrollment, and the study was approved by the Research Ethics Committee of Ren Ji Hospital, School of Medicine, Shanghai Jiao Tong University.

\section{Cell culture and reagent}

Human PDAC cell lines AsPC1, BxPC-3, CFPAC-1, HPAC, PANC1 and SW1990 were all preserved in Shanghai Cancer Institute, Ren Ji Hospital, School of Medicine, Shanghai Jiao Tong University and normal human pancreatic ductal cell line hTERT-HPNE was purchased from American Type Culture Collection (ATCC, Manassas, VA). All of these cells were cultured in indicated medium according to ATCC protocols, and supplemented with $10 \%(\mathrm{v} / \mathrm{v})$ fetal bovine serum (FBS) and $1 \%$ antibiotics $(100 \mu \mathrm{g} / \mathrm{ml}$ streptomycin and 100 units/ $\mathrm{ml}$ penicillin) at $37^{\circ} \mathrm{C}$ in a humidified incubator under $5 \% \mathrm{CO}_{2}$ condition. Human umbilical vein endothelial cells (HUVECs) were a generous gift from Dr. Huan Yi (Shanghai No.5 People's Hospital, Fudan University). ABT-199 was purchased from Selleck (Shanghai, China).

\section{Immunohistochemical staining}

Immunohistochemical (IHC) staining was performed as previously described [36]. Briefly, after tissue sections were deparaffinized, rehydrated with graded ethanol, incubated with $0.3 \%$ hydrogen peroxide for 30 minutes and blocked with 10\% BSA (Sangon, Shanghai, China), slides were first incubated using the antibody for EDIL3 (dilution 1:200, Proteintech, US), PCNA (dilution 1:5000, CST, US), cleaved caspase 3 (dilution 1:2000, CST, US) and Bcl-2 (dilution 1:300, Proteintech, US) at $4^{\circ} \mathrm{C}$ overnight, labeled by HRP (rabbit) second antibody (Thermo Scientific, US) at room temperature for $1 \mathrm{~h}$. Finally, positive staining was visualized with DAB substrate liquid (Gene Tech, Shanghai), and counterstained by hematoxylin. All the sections were observed and photographed with a microscope (Carl Zeiss, Germany). Scoring was conducted according to the ratio of positivestaining cells: $0-5 \%$ scored $0 ; 6-35 \%$ scored $1 ; 36-70 \%$ scored 2 ; more than $70 \%$ scored 3 and staining intensity: no staining scored 0 , weakly staining scored 1 , moderately staining scored 2 and strongly staining scored 3 . The final score was designated using the percent of positive cell score $\times$ staining intensity score as follows: "-" for a score of $0-1$, " "+" for a score of 2-3, "++" for a score of 4-6 and " +++ " for a score of $>6$; low expression was defined as a total score $<4$ and high expression with a total score $\geq 4$. These scores were determined independently by two senior pathologists in a blinded manner.

\section{Quantitative real-time PCR}

Total RNA was extracted from primary tumor and corresponding non-cancerous using Trizol reagent (Takara,
Japan), and reversely transcribed through PrimeScript RT-PCR kit (Takara, Japan) according to the manufacturer's instructions. Quantitative real-time PCR was performed with SYBR Premix Ex Taq (Takara, Japan) on a 7500 Real-time PCR system (Applied Biosystems, Inc. USA). Primer sequences used in this study were as follows: EDIL3, forward 5'-AGCATACCGAG GGGATACATT-3', reverse 5'-CAAGGCTCAACTTCGC ATTCA-3'; $\beta$-actin , forward 5'-ACTCGTCATACTCC TGCT-3', reverse 5'-GAAACTACCTTCAACTCC-3'. The $2^{-\Delta \mathrm{Ct}}$ method was used to quantify the relative EDIL3 expression levels and normalized using the $\beta$-actin expression.

\section{Establishment of stable EDIL3 knockdown cell lines}

Short hairpin RNA (shRNA)-containing plasmids were packaged into lenti-virus and virus titers were determined. Two target cell lines, SW1990 and BxPC-3, were infected with $1 \times 10^{6}$ recombinant lentivirus-transducing units in the presence of 6 $\mu \mathrm{g} / \mathrm{ml}$ polybrene (Sigma, Shanghai, China). The sequences targeting EDIL3 are as follows; sh-1: 5'-CCGGGGAGGTTGCATCAGATGAAGACTCGAGT CTTCATCTGATGCAACCTCCTTTTTG-3'; sh-2: 5'CCGGGGGTATGAAATCAGGACATATCTCGAGA TATGTCCTGATTTCATACCCTTTTTG-3'. The stable EDIL3 knockdown cells were selected in the presence of $2 \mu \mathrm{g} / \mathrm{ml}$ puromycin. The knockdown efficacy was tested by western blotting.

\section{Western blotting}

Cell total protein was extracted using a total protein extraction buffer (Beyotime, China) and the protein concentration was measured using a BCA Protein Assay Kit (Pierce Biotechnology). Cell lysates were separated by $8-12 \%$ SDS-PAGE gel electrophoresis and transferred to a PVDF membrane. After blocking with $1 \%$ BSA, the membrane was probed with one of the following primary antibodies: EDIL3, Bcl-2, Bcl-xL, Bax, $\beta$-actin (Proteintech, US) and species-specific secondary antibodies. Bound secondary antibodies were detected by Odyssey imaging system (LI-COR Biosciences, Lincoln, NE).

\section{Cell viability assay}

Recombinant EDIL3 expression, purification and characterization were performed as previously described [15]. For cell viability assay, cells were seeded into a 96well plate at $3 \times 10^{3}$ cells per well with $100 \mu$ culture medium and cultured at $37^{\circ} \mathrm{C}$. The cell viability was quantified by Cell Counting Kit-8 (CCK-8, Dojindo, Japan). Briefly, by addition of $10 \%$ (v/v) CCK-8 to the culture medium and incubation for $1 \mathrm{~h}$, cell viability 
was monitored by measuring absorbance at $450 \mathrm{~nm}$ using a Power Wave XS microplate reader (BIO-TEK). The experiment was performed in quintuplicate and repeated twice.

\section{Cell migration and invasion assay}

The invasive potential of PDAC cells was measured by transwell model (Corning, NY, USA) according to the manufacturer's instructions. For migration assay, $2 \times 10^{4}$ cells in $100 \mu \mathrm{l}$ medium were seeded into the upper chamber of the transwell inserts. The invasion assay was performed with matrigel-coated filters (BD Bioscience, USA). RPMI 1640 or DMEM medium containing 5 $\%(\mathrm{v} / \mathrm{v}) \mathrm{FBS}$ and recombinant EDIL3 was added to the bottom chamber. Cells were incubated at $37^{\circ} \mathrm{C}$ and allowed to migrate for $24 \mathrm{~h}$ or invade through Matrigel for $48 \mathrm{~h}$. At the designated time points, the non-invading cells that remained on the upper surface were removed. The migrated and invaded cells were fixed with $4 \%$ paraformaldehyde and stained with $0.1 \%$ crystal violet. The number of cells on the lower surface was counted under a light microscope in six random fields. Each experiment was performed in triplicate and repeated twice.

\section{Apoptosis and anoikis assay}

For cell apoptosis assay, $20 \times 10^{5}$ cells per well in the presence or absence of recombinant EDIL3 treatment were cultured under serum-deprivation in 6-well plates. Adherent cells were detached with $0.25 \%$ trypsin without EDTA in $1 \times$ PBS. Cells were harvested in complete RPMI 1640 or DMEM medium and centrifuged at 1000 rpm for $5 \mathrm{~min}$. Each of the cells were washed with $1 \times$ PBS, stained with $50 \mu \mathrm{g} / \mathrm{ml}$ propidium iodide (PI) and Annexin V-FITC (BD Pharmingen, USA) following the manufacturer's instructions. The percentage of Annexin $\mathrm{V}(+)$ and PI (-) cells were analyzed by flow cytometry. For anoikis assay, Annexin V/PI staining and Caspase-3/7 activity (Promega, USA) were performed as previously described [15].

\section{Colony formation assay}

Colony formation in soft agar was performed to evaluate anchorage-independent growth. A total of $1.0 \mathrm{ml}$ of RPMI 1640 or DMEM medium containing 10\% FBS and $0.5 \%$ agar was plated in the bottom of 6 -well plates. PANC1 or AsPC1 cells at $2 \times 10^{3}$ cells per well were suspended in the upper layer consisted of $1 \%$ FBS culture medium and $0.35 \%$ agar. For knockdown assay, cells were fed every 2 days with complete medium. For EDIL3 stimulation assay, the soft agars were fed every 2 days with serum-free culture medium in the presence or absence of recombinant EDIL3 at indicated concentrations. After
14-21 days, colonies were stained with $0.05 \%$ crystal violet, and all the visible colonies were counted by microscopy.

\section{Animal experiments}

Mice were manipulated and housed according to protocols approved by the East China Normal University Animal Care Commission. All animals received humane care according to the criteria outlined in the "Guide for the Care and Use of Laboratory Animals" prepared by the National Academy of Sciences and published by the National Institutes of Health. Athymic male NU/NU mice ages 6 weeks were kept on a 12-hour day/night cycle with free access to food and water. SW1990 cells were trypsinized (Invitrogen, USA), washed in PBS, and resuspended in serum-free DMEM. A total of $3 \times 10^{6}$ SW1990 cells in 200 ul DMEM medium were injected subcutaneously in the lower back. Tumor growth was monitored after three weeks after mouse were sacrificed.

\section{Statistical analysis}

Data were presented as the means \pm SD. The SPSS software program (version 17.0; IBM Corporation) was used for statistical analysis. Graphical representations were performed with GraphPad Prism 5 (San Diego, CA) software. Correlation of EDIL3 expression with clinicopathologic parameters in patients with PDAC was evaluated by chi-square test or Fisher's exact test. Survival curves were evaluated using the Kaplan-Meier method and differences between survival curves were tested by the log-rank test. Univariate and multivariate Cox regression analyses were performed to identify the factors that had a significant influence on survival by Cox proportional hazards model. The student's $t$-test or one-way ANOVA was used for comparison between groups. $P<0.05$ was considered statistically significant.

\section{ACKNOWLEDGMENTS}

This work was supported by grants from National High Technology Research and Development Program of China (no.2014AA020609), State Key Laboratory for Oncogenes and Related Genes (YSJJ-2014-01) and additional grants from Shanghai Key Medical Specialty Program (ZK2012A22).

\section{CONFLICTS OF INTEREST}

The authors have no conflicts of interest.

\section{REFERENCES}

1. Vincent A, Herman J, Schulick R, Hruban RH, Goggins M. Pancreatic cancer. Lancet. 2011; 378:607-620. 
2. Siegel R, Naishadham D, Jemal A. Cancer statistics, 2012.CA Cancer J Clin. 2012; 62:10-29.

3. The Lancet O. Pancreatic cancer in the spotlight. Lancet Oncol. 2014; 15:241.

4. Pliarchopoulou K, Pectasides D. Pancreatic cancer: current and future treatment strategies. Cancer Treat Rev. 2009; 35:431-436.

5. Stathis A, Moore MJ. Advanced pancreatic carcinoma: current treatment and future challenges. Nat Rev Clin Oncol. 2010; 7:163-172.

6. Kim SH, Turnbull J, Guimond S. Extracellular matrix and cell signalling: the dynamic cooperation of integrin, proteoglycan and growth factor receptor. J Endocrinol. 2011; 209:139-151.

7. Hidai C, Zupancic T, Penta K, Mikhail A, Kawana M, Quertermous EE, Aoka Y, Fukagawa M, Matsui Y, Platika D, Auerbach R, Hogan BL, Snodgrass R and Quertermous T. Cloning and characterization of developmental endothelial locus-1: an embryonic endothelial cell protein that binds the alphavbeta3 integrin receptor. Genes Dev. 1998; 12:21-33.

8. Choi EY. Inhibition of leukocyte adhesion by developmental endothelial locus-1 (del-1). Immune Netw. 2009; 9:153-157.

9. Rezaee M, Penta K, Quertermous T. Del1 mediates VSMC adhesion, migration, and proliferation through interaction with integrin alpha(v)beta. Am J Physiol Heart Circ Physiol. 2002; 282:H1924-1932.

10. Aoka Y, Johnson FL, Penta K, Hirata Ki K, Hidai C, Schatzman R, Varner JA, Quertermous T. The embryonic angiogenic factor Dell accelerates tumor growth by enhancing vascular formation. Microvasc Res. 2002; 64:148-161.

11. Lee JE, Moon PG, Cho YE, Kim YB, Kim IS, Park H, Baek MC. Identification of EDIL3 on extracellular vesicles involved in breast cancer cell invasion. J Proteomics. 2015.

12. Zou X, Qiao H, Jiang X, Dong X, Jiang H, Sun X. Downregulation of developmentally regulated endothelial cell locus-1 inhibits the growth of colon cancer. J Biomed Sci. 2009; 16:33.

13. Beckham CJ, Olsen J, Yin PN, Wu CH, Ting HJ, Hagen FK, Scosyrev E, Messing EM, Lee YF. Bladder cancer exosomes contain EDIL-3/Del1 and facilitate cancer progression. J Urol. 2014; 192:583-592.

14. Sun JC, Liang XT, Pan K, Wang H, Zhao JJ, Li JJ, Ma HQ, Chen YB, Xia JC. High expression level of EDIL3 in HCC predicts poor prognosis of HCC patients. World J Gastroenterol. 2010; 16:4611-4615.

15. Feng MX, Ma MZ, Fu Y, Li J, Wang T, Xue F, Zhang JJ, Qin WX, Gu JR, Zhang ZG, Xia Q. Elevated autocrine EDIL3 protects hepatocellular carcinoma from anoikis through RGD-mediated integrin activation. Mol Cancer. 2014; 13:226.

16. Xia H, Jianxiang C, Shi M, Gao H, Karthik S, Pratap SV, Ooi LL, Hui KM. EDIL3 is a novel regulator of epithelial mesenchymal transition controlling early recurrence of hepatocellular carcinoma. J Hepatol. 2015.
17. Bergers G, Javaherian K, Lo KM, Folkman J, Hanahan D. Effects of angiogenesis inhibitors on multistage carcinogenesis in mice. Science. 1999; 284:808-812.

18. Ferrara N, Kerbel RS. Angiogenesis as a therapeutic target. Nature. 2005; 438:967-974.

19. Chiarugi P, Giannoni E. Anoikis: a necessary death program for anchorage-dependent cells. Biochem Pharmacol. 2008; 76:1352-1364.

20. Tan K, Goldstein D, Crowe P, Yang JL. Uncovering a key to the process of metastasis in human cancers: a review of critical regulators of anoikis. J Cancer Res Clin Oncol. 2013; 139:1795-1805.

21. Pollman MJ, Naumovski L, Gibbons GH. Endothelial cell apoptosis in capillary network remodeling. J Cell Physiol. 1999; 178:359-370.

22. Oltersdorf T, Elmore SW, Shoemaker AR, Armstrong RC, Augeri DJ, Belli BA, Bruncko M, Deckwerth TL, Dinges J, Hajduk PJ, Joseph MK, Kitada S, Korsmeyer SJ, et al. An inhibitor of Bcl-2 family proteins induces regression of solid tumours. Nature. 2005; 435:677-681.

23. Hardwick JM, Youle RJ. SnapShot: BCL-2 proteins. Cell. 2009; 138:404, 404 e401.

24. Choi EY, Chavakis E, Czabanka MA, Langer HF, Fraemohs L, Economopoulou M, Kundu RK, Orlandi A, Zheng YY, Prieto DA, Ballantyne CM, Constant SL, Aird WC, et al. Del-1, an endogenous leukocyte-endothelial adhesion inhibitor, limits inflammatory cell recruitment. Science. 2008; 322:1101-1104.

25. Dasgupta SK, Le A, Chavakis T, Rumbaut RE, Thiagarajan P. Developmental endothelial locus-1 (Del-1) mediates clearance of platelet microparticles by the endothelium. Circulation. 2012; 125:1664-1672.

26. Hanayama R, Tanaka M, Miwa K, Nagata S. Expression of developmental endothelial locus-1 in a subset of macrophages for engulfment of apoptotic cells. J Immunol. 2004; 172:3876-3882.

27. Kim H, Lee SH, Lee MN, Oh GT, Choi KC, Choi EY. p53 regulates the transcription of the anti-inflammatory molecule developmental endothelial locus-1 (Del-1). Oncotarget. 2013; 4:1976-1985. doi: 10.18632/oncotarget.1318.

28. Aoki M, Kanamori M, Ohmori K, Takaishi M, Huh NH, Nogami S, Kimura T. Expression of developmentally regulated endothelial cell locus 1 was induced by tumorderived factors including VEGF. Biochem Biophys Res Commun. 2005; 333:990-995.

29. Cabebe E, Fisher GA. Clinical trials of VEGF receptor tyrosine kinase inhibitors in pancreatic cancer. Expert Opin Investig Drugs. 2007; 16:467-476.

30. Buchler P, Reber HA, Buchler MW, Friess H, Hines OJ. VEGF-RII influences the prognosis of pancreatic cancer. Ann Surg. 2002; 236:738-749; discussion 749.

31. Weissmueller S, Manchado E, Saborowski M, Morris JPt, Wagenblast E, Davis CA, Moon SH, Pfister NT, Tschaharganeh DF, Kitzing T, Aust D, Markert EK, et al. 
Mutant p53 drives pancreatic cancer metastasis through cell-autonomous PDGF receptor beta signaling. Cell. 2014; 157:382-394.

32. Iacobuzio-Donahue CA, Herman JM. Autophagy, p53, pancreatic cancer. N Engl J Med. 2014; 370:1352-1353.

33. Morton JP, Timpson P, Karim SA, Ridgway RA, Athineos D, Doyle B, Jamieson NB, Oien KA, Lowy AM, Brunton VG, Frame MC, Evans TR, et al. Mutant p53 drives metastasis and overcomes growth arrest/senescence in pancreatic cancer. Proc Natl Acad Sci U S A. 2010; 107:246-251.

34. Damhofer H, Medema JP, Veenstra VL, Badea L, Popescu I, Roelink H, Bijlsma MF. Assessment of the stromal contribution to Sonic Hedgehog-dependent pancreatic adenocarcinoma. Mol Oncol. 2013; 7:1031-1042.

35. Penta K, Varner JA, Liaw L, Hidai C, Schatzman R, Quertermous T. Dell induces integrin signaling and angiogenesis by ligation of alphaVbeta3. J Biol Chem. 1999; 274:11101-11109.

36. Jiang SH, He P, Ma MZ, Wang Y, Li RK, Fang F, Fu Y, Tian GA, Qin WX, Zhang ZG. PNMA1 promotes cell growth in human pancreatic ductal adenocarcinoma. Int J Clin Exp Pathol. 2014; 7:3827-3835. 